\title{
Peritonitis tuberculosa en un niño
}

\author{
Daniela Arriagada ${ }^{1}$, Megan Neumann ${ }^{2}$ y Alejandro Donoso ${ }^{1}$
}

\footnotetext{
\Unidad de Paciente Crítico Pediátrico. Hospital Clínico Dra. Eloísa Díaz I. La Florida. Santiago,

EEscuela de Medicina. Universidad Andrés Bello. Santiago, Chile.

Recibido: 30 de abril de 2019 Aceptado: 24 de octubre de 2019

Correspondencia a: Alejandro Donoso F. adonosofuentes@gmail.com
}

\section{Pediatric tuberculous peritonitis. Case report}

Tuberculous peritonitis is an uncommon entity in the infant population. It is an uncommon form of extrapulmonary tuberculosis and represents a very low percentage of all cases of tuberculosis. Its symptoms are nonspecific and usually manifesting with ascites, abdominal pain, fever and low weight. The delay in its diagnosis and treatment, originated by its form of presentation, can cause an increase in its morbidity and mortality. We report the case of a 14-year-old patient without concomitant disease or pulmonary tuberculosis, who presented with ascites and fever. Laparoscopy showed multiple nodules in the abdominal cavity compatible with peritoneal tuberculosis, which was subsequently confirmed by culture and molecular test. The patient completed her antituberculosis treatment recovering satisfactorily.

Keywords: Tuberculosis; tuberculous peritonitis; Mycobacterium tuberculosis; ascites.

Palabras clave: Tuberculosis; peritonitis tuberculosa; Mycobacterium tuberculosis; ascitis.

\section{Introducción}

$\mathrm{L}$ a tuberculosis (TBC) continúa siendo una de las diez primeras causas de muerte a nivel mundial y la primera por enfermedades infecciosas.

En Chile, la incidencia de esta enfermedad es una de las menores de Latinoamérica, y es más baja aún en los menores de 15 años ${ }^{1}$. Del análisis del registro nacional del programa, en el último lustro se han producido 255 casos de TBC en menores de 15 años, con una leve preponderancia en las mujeres. El factor de riesgo más importante para la TBC infantil es ser contacto de un caso bacilífero intradomiciliario, antecedente que está presente en un tercio de los casos ${ }^{2}$.

De todos los sitios afectados por la TBC extrapulmonar, el compromiso abdominal es el sexto más frecuente ${ }^{3}$. Ocurre en aproximadamente $11 \%$ de los casos y puede comprometer el peritoneo, tracto gastrointestinal, linfonodos mesentéricos o una víscera sólida ${ }^{4}$. En niños la localización más frecuente es el peritoneo y linfonodos ${ }^{5,6}$.

La TBC peritoneal constituye menos de $1 \%$ de todos los casos de $\mathrm{TBC}^{7}, 4-10 \%$ de la TBC extrapulmonar ${ }^{8} \mathrm{y}$ $25-60 \%$ de los casos de TBC abdominal ${ }^{9}$. Se presenta con mayor frecuencia en adultos entre los 35-45 años ${ }^{7,10}$, siendo reportada muy ocasionalmente en niños ${ }^{6,11}$, con una incidencia de $0,3 \%$ en E.U.A en menores de 20 años ${ }^{5}$. Su sintomatología es inespecífica por lo que su diagnóstico es difícil de confirmar y puede imitar otras enfermedades abdominales (inflamatorias, tumorales) ${ }^{10,12}$.

Enfermedades asociadas como cirrosis hepática, infección por VIH, cáncer, tratamiento inmunosupresor, diálisis peritoneal y población inmigrante originaria de regiones con alta prevalencia ${ }^{13}$, tienen riesgo aumentado de TBC peritoneal. No obstante, es aun de rara ocurrencia en nuestro país.

Se presenta el caso de una adolescente de 14 años, sin enfermedad concomitante o TBC pulmonar en quién se diagnosticó una TBC peritoneal.

\section{Caso clínico}

Adolescente chilena de 14 años, con ambos padres sanos y esquema de inmunización completa. Con antecedentes de asma bronquial tratado hasta los 12 años. Sin antecedentes familiares ni contactos con pacientes con TBC o tosedores crónicos. Consumo ocasional de productos lácteos no pasteurizados.

Ingresó a la unidad de cuidados intermedios por historia de cuatro meses de fiebre, disminución de peso $(6 \mathrm{~kg})$, dolor y distensión abdominal con episodios alternantes de diarrea y períodos de constipación. Ingresó afebril con hemodinamia estable. Se encontraba enflaquecida, con IMC: $18,1 \mathrm{~kg} / \mathrm{m}^{2}(-1 \mathrm{DE})$, perímetro abdominal de $72 \mathrm{~cm}$ y abdomen sensible, con una masa palpable en hemiabdomen inferior. Resto del examen segmentario normal. Hemograma con hematocrito $31,6 \%$, hemoglobina $10 \mathrm{~g} / \mathrm{dl}$, leucocitos $5.900 / \mathrm{mm}^{3}$ (74 \% segmentados), PCR 4,13 mg/dl (VN $<1 \mathrm{mg} / \mathrm{dl})$. La ecografía abdominal reveló una imagen anecogénica tabicada que se extendía desde región anexial izquierda hacia el cuadrante superior derecho, desplazando asas intestinales hacia posterior. Se complementó el estudio con una tomografía computarizada (TC) de abdomen y pelvis que evidenció una 
colección abdominal de 29 × 17 × $8 \mathrm{~cm}$ que contactaba con ovario izquierdo, con realce del peritoneo parietal $\mathrm{y}$ asas intestinales, líquido libre laminar hiperdenso y numerosas adenopatías mesentéricas hasta $14 \mathrm{~mm}$ de diámetro (Figura 1).

Se solicitaron marcadores tumorales destacando CA125: $236 \mathrm{U} / \mathrm{ml}$ (VN 0-35 U/ml). Antígeno carcinoembrionario, gonadotrofina coriónica humana y alfa-fetoproteína estaban en rangos normales. Por hallazgos en TC y CA-125 elevado se realizó una ecografía ginecológica que descartó patología anexial.

Se inició terapia antimicrobiana triasociada de amplio espectro y se realizó una laparoscopia exploradora. Se visualizó gran cantidad de líquido peritoneal de aspecto citrino y existencia de múltiples adherencias laxas que formaban loculaciones. La superficie peritoneal y diafragmática tenía presencia de múltiples lesiones blanquecinas de aspecto miliar ("granos de mijo"). El líquido peritoneal fue levemente opalescente, con glucosa $22 \mathrm{mg} / \mathrm{dl}$, proteínas $5.090 \mathrm{mg} / \mathrm{dl}$, leucocitos 40 céls $/ \mathrm{mm}^{3}$, $100 \%$ mononucleares, y hematíes 120 céls $/ \mathrm{mm}^{3}$. En la tinción de Gram no se observaron bacterias. En el estudio microbiológico destacó una RPC para complejo Mycobacterium tuberculosis de líquido peritoneal positiva. $\mathrm{La}$ baciloscopia de líquido peritoneal fue negativa para bacilos ácido-alcohol resistentes (BAAR). La determinación de QuantiFERON TB Gold ${ }^{\text {ò }}$ In Tube fue positiva $(0,53$ $\mathrm{UI} / \mathrm{ml}$ ) y la prueba cutánea de tuberculina (PPD) fue 22 $\mathrm{mm}$. El cultivo de Koch en líquido peritoneal fue positivo para $M$. tuberculosis sensible a rifampicina e isoniacida.

Ante estos hallazgos, se inició un esquema primario de terapia antituberculosa en dosis única combinada con una duración total de seis meses. Completó la fase diaria de 50 dosis, con rifampicina $150 \mathrm{mg}$, isoniacida $75 \mathrm{mg}$, pirazinamida $400 \mathrm{mg}$ y etambutol $275 \mathrm{mg}$ y la fase trisemanal de 48 dosis con rifampicina $150 \mathrm{mg}$ e isoniacida $150 \mathrm{mg}$, según el protocolo vigente.

La biopsia de tejido peritoneal demostró tejido conectivo con un proceso inflamatorio crónico granulomatoso necrosante, de tipo tuberculoideo. Se observaron células gigantes multinucleadas de tipo Langhans, con tinción de Ziehl-Neelsen negativa para BAAR.

Dentro de la evaluación para descartar compromiso sistémico, se realizó una punción lumbar, con una RPC de LCR negativa para $M$. tuberculosis y adenosindeaminasa (ADA) normal. La TC de cerebro no tenía hallazgos patológicos. La baciloscopia de esputo y contenido gástrico fueron negativas. La radiografía y TC de tórax no tenían hallazgos patológicos (Figura 2). La ecocardiografía y radiografía de columna total y pelvis fueron normales. Se realizó un estudio inmunológico básico (niveles de inmunoglobulinas séricas y cuantificación de complemento C3

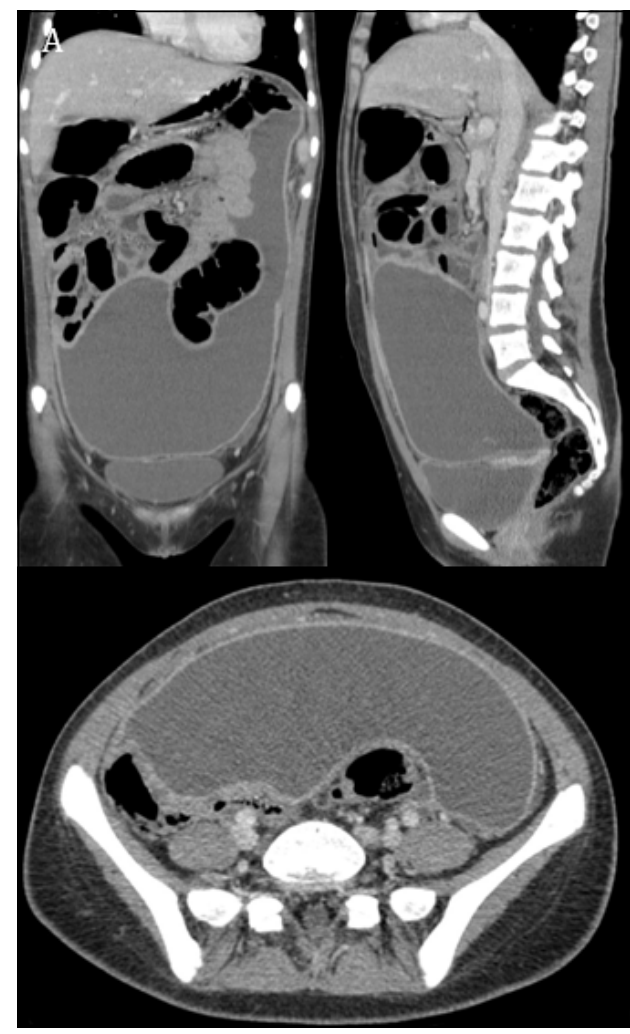

B

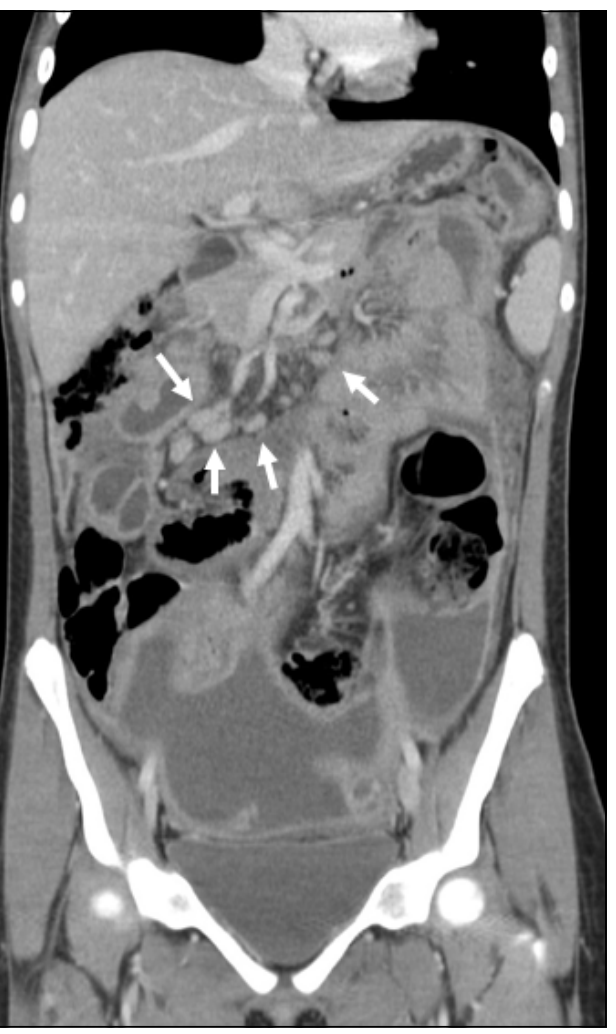

Figura 1. Tomografía computarizada de abdomen. A. Extensa colección centrada a nivel del hemiabdomen inferior. Engrosamiento del plano peritoneal el cual realza tras la administración de medio de contraste. B. Múltiples adenopatías mesentéricas de aspecto reactivo (flechas blancas). 


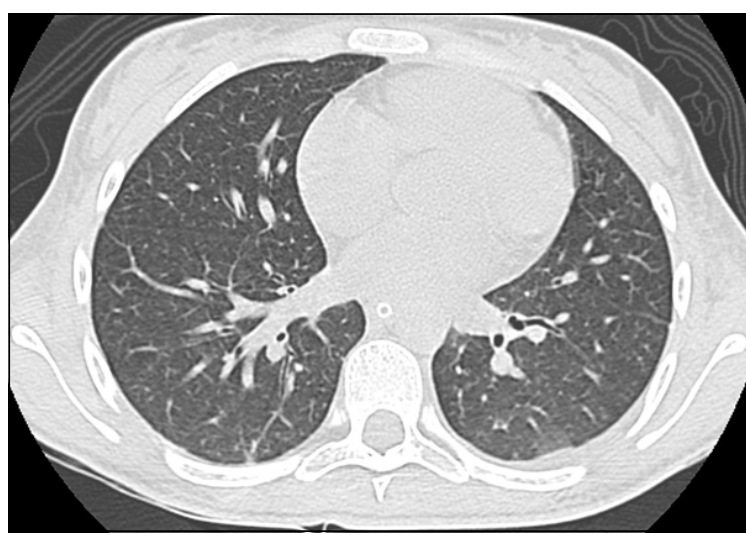

Figura 2. Tomografía computarizada tórax en la cual no se observan imágenes sugerentes de compromiso pulmonar.

y C4) que fue normal, con serología para VIH negativa.

El estudio familiar evidenció un PPD positivo en un hermano de 15 años $(15 \mathrm{~mm})$, sobrino de 5 años $(15 \mathrm{~mm})$ y primo de 6 años $(14 \mathrm{~mm})$, motivo por lo cual se les inició quimioprofilaxis con isoniacida por seis meses.

Luego de dos semanas de hospitalización la paciente fue dada de alta en buenas condiciones generales, afebril, sin dolor abdominal ni ascitis. Una ecografía abdominal realizada a los nueve meses del egreso no tuvo hallazgos patológicos. El seguimiento a los tres años de evolución, se encontraba en buenas condiciones, sin síntomas abdominales y con buen incremento ponderal.

\section{Discusión}

La TBC se presenta muy ocasionalmente en niños. Menos de $5 \%$ del total ocurren en menores de 14 años ${ }^{14}$, con solo algunos casos descritos en lactantes. Los pacientes de menor edad han sido lactantes de 6 y 8 meses, quienes se presentaron con fiebre, vómitos y distensión abdominal ${ }^{15}$. En ambos, se realizó laparotomía exploradora evidenciándose ascitis, adherencias intestinales y granulaciones ${ }^{15}$.

En la mayoría de los casos pediátricos de TBC peritoneal existe historia familiar de infección activa ${ }^{16}$, hallazgo comunicado hasta en $60 \%$ de los $\operatorname{casos}^{11,17}$. Asimismo, las manifestaciones extrapulmonares de la TBC son más comunes en pacientes inmunocomprometidos ${ }^{13}$.

Se han postulado diferentes mecanismos por el cual ocurre el compromiso peritoneal, tal como diseminación hematógena por reactivación de un foco pulmonar primario, aunque también puede ocurrir durante una TBC pulmonar activa o TBC miliar ${ }^{18}$. Se ha descrito, mucho menos frecuentemente, por acceso directo desde un linfonodo mesentérico infectado, mediante diseminación transmural desde el intestino delgado o por contigüidad desde una salpingitis tuberculosa. Una proporción signifi- cativa de pacientes ( 15 a $20 \%$ ) con TBC abdominal tiene una enfermedad pulmonar activa ${ }^{19}$.

Generalmente su comienzo es insidioso; más de $70 \%$ de los pacientes presentaron síntomas por más de cuatro meses $^{20}$. El espectro clínico no exhibe características específicas, dificultando el diagnóstico. Los síntomas y signos son principalmente constitucionales, siendo los más comunes ascitis $(73 \%)$, dolor abdominal $(65 \%)$, pérdida de peso $(61 \%)$, fiebre $(59 \%)$, diarrea $(21 \%)$ y constipación $(11 \%)^{21}$. Esporádicamente se presenta como una obstrucción intestinal ${ }^{22}$, abdomen agudo ${ }^{23}$ o TBC grave diseminada (Sepsis tuberculosa gravissima $)^{24}$.

El líquido ascítico es característicamente un exudado de predominio linfocítico con una gradiente de albúmina sérica/ascitis $<1,1 \mathrm{~g} / \mathrm{d}^{25}$. La radiografia de tórax puede ser anormal entre 19 y $83 \%$ de los $\operatorname{casos}^{19}$. Un estudio con diez pacientes (promedio de edad de 14 años) mostró alteraciones radiológicas en $90 \%$ de los pacientes ${ }^{17}$, mientras que Dinler y cols. ${ }^{16}$ las encontraron entre $50-75 \%$ de los pacientes, y de estos 12 a $63 \%$ presentaban también derrame pleural. En contraparte, en otra serie se comunicaron imágenes pulmonares de TBC activa o secuelar en sólo un tercio de los pacientes ${ }^{13}$. Por esta razón, la ausencia de hallazgos en la imagenología de tórax no descarta una posible infección extrapulmonar ${ }^{13,26}$.

La elevación antígeno del cáncer 125 (CA-125) se ha documentado en la mayoría de los pacientes con TBC peritoneal, elevándose hasta diez veces su valor. Simsek y cols. ${ }^{27}$ realizaron un estudio de casos y controles encontrando elevación del CA-125 en todos los pacientes con TBC peritoneal. Sin embargo, se debe considerar que esto ocurre en cualquier ascitis de carácter exudativo ${ }^{28}$, por lo que carece de especificidad. No obstante, los valores de este marcador pueden ser útiles para evaluar la eficacia del tratamiento antituberculoso ${ }^{29}$ ya que declina rápidamente, en paralelo, con la respuesta clínica y resolución de la ascitis?

La prueba de tuberculina (purified proteic derivate, PPD), tiene un valor limitado por su baja especificidad ${ }^{30}$, como también menor sensibilidad en el contexto clínico de inmunosupresión ${ }^{31} \mathrm{y}$ en las formas extrapulmonares; finalmente un resultado negativo no descarta la infección ${ }^{32}$.

El uso de estudios complementarios de imagenología, que incluye la ecografía y la TC abdominal contrastada, son importantes en el diagnóstico de la TBC peritoneal. En la TC abdominal los principales signos sugerentes son la presencia de ascitis de alta densidad con o sin tabiques, engrosamiento del epiplón, peritoneo y mesenterio, existencia de ganglios linfáticos hipodensos en su centro y aglutinación de asas intestinales ${ }^{33}$, mientras que la ecografía abdominal, aunque es poco específica en cuanto a sus hallazgos, permite observar mejor las pequeñas loculaciones peritoneales ${ }^{7}$.

Las técnicas de diagnóstico inmunológico pertene- 
cientes al grupo de las llamadas pruebas de liberación de interferón-gamma (IF-g) (Interferon Gamma Release Assays, IGRA) como QuantiFERON TB Gold ${ }^{\circledR}$ In Tube y el T-SPOT ${ }^{\circledR}$ TB cuantifican in vitro la concentración de IF-g y el número de células que lo liberan; respectivamente, correspondiendo a la respuesta inmune celular del linfocito T sensibilizado por antígenos específicos de $M$. tuberculosi $i^{34}$. Aunque es una modalidad diagnóstica de gran utilidad, existe poca información en pediatría, particularmente en menores de 5 años de edad ${ }^{31,34-36}$. Empero, un reciente trabajo ha demostrado una alta sensibilidad y especificidad en pacientes con TBC latente, especialmente en menores de 5 años ${ }^{35}$.

Recientemente, técnicas de RPC modificada (Técnica Xpert MTB/RIF, RPC en tiempo real o RPC anidada) han sido utilizadas para la detección precoz de Mycobacterium en líquido ascítico, sin embargo, los resultados no son concluyentes $^{37,38}$. La técnica GeneXpert MTB/RIF es rápida y eficiente pudiendo detectar en forma simultánea M. tuberculosis como resistencia a la rifampicina. Esta prueba está recomendada para muestras pulmonares con valores de sensibilidad y especificidad globales de 88 y $99 \%$; respectivamente y para la resistencia a rifampicina se reporta una sensibilidad de $95 \%$ y especificidad de $98 \%{ }^{39}$. Sin embargo, su utilidad para el diagnóstico de TBC extrapulmonar, particularmente en líquido ascítico es comparativamente débil ${ }^{40}$. Rufai y cols. ${ }^{37}$ evaluaron su sensibilidad utilizando muestras de liquido ascítico (considerando el MGIT-960 como estándar de oro). De 67 pacientes solo resultó positivo en 17,9\% de los casos. Asimismo, su rendimiento diagnóstico fue bajo, incluso en cultivos positivos (70,5\%). En otro estudio, la sensibilidad del GeneXpert para el diagnóstico de TBC abdominal fue $28,5 \%$, lo cual fue similar a lo reportado por Alvarez-Uria y cols. ${ }^{42}$ con una sensibilidad de $27,8 \%$ en muestra de líquido ascítico. No obstante, a pesar de su baja sensibilidad, posee un alto valor predictivo positivo, por lo que el tratamiento debe iniciarse sin esperar otros resultados, si este resulta positivo ${ }^{37,41}$.

Finalmente, la laparoscopia con biopsia peritoneal dirigida presenta una sensibilidad por sobre $90 \%{ }^{43}$. Como en el caso aquí comunicado, en los hallazgos laparoscópicos en más del $90 \%$ de los casos se visualizan numerosos nódulos blanquecinos-amarillentos $(<5 \mathrm{~mm})$ dispersos por un engrosado peritoneo visceral y parietal, con múltiples adherencias. En el estudio histológico es posible encontrar una inflamación de carácter granulomatosa necrosante de tipo tuberculoide.

La medición de la actividad de la adenosinadesaminasa (ADA) en el líquido ascítico se ha empleado como técnica no invasiva y rápidamente disponible. Este marcador de respuesta inflamatoria e inmunitaria es altamente sensible (100\%) y específico (97\%) para esta infección ${ }^{6,20}$.
La identificación microbiológica de M. tuberculosis constituyen el estándar de oro para el diagnóstico definitivo $^{20}$. Sin embargo, el aislamiento bacteriano tiene un pobre rendimiento para la TBC peritoneal ${ }^{16}$. La tinción para BAAR y el cultivo tienen baja sensibilidad, reportándose $3 \%$ para la tinción ${ }^{7}$ y sólo $35 \%$ para el cultivo ${ }^{7}$. El rendimiento puede aumentar cuando se cultivan grandes volúmenes de líquido ascítico. Singh y cols. ${ }^{44}$ comunicaron una tasa de positividad de $83 \%$ para el cultivo de un litro de líquido peritoneal. Su uso para el diagnóstico es difícil, ya que el crecimiento bacteriano requiere entre 4-8 semanas $^{53}$, por lo tanto, la mortalidad asociada a TBC peritoneal es elevada entre los pacientes que esperan por el resultado microbiológico ${ }^{9,13}$. No obstante, el uso del sistema radiométrico BACTEC ${ }^{\circledR}$ ha disminuido este tiempo a dos semanas ${ }^{19}$. Por consiguiente, la falta del agente patógeno no elimina la posibilidad de la enfermedad, motivo por el cual es importante el uso de métodos diagnósticos complementarios. Actualmente el estudio molecular y la transcriptómica son otras opciones en pleno desarrollo ${ }^{45}$.

Su diagnóstico diferencial debe ser necesariamente con otras enfermedades infecciosas, enfermedades inflamatorias intestinales y neoplasias (esta última especialmente en mujeres $)^{46}$. Recientemente se ha comunicado la utilidad del empleo del PET/TC (tomografía por emisión de positrones - tomografía computada) en el peritoneo parietal para diferenciar con carcinomatosis peritoneal ${ }^{47}$.

De acuerdo con la norma ministerial nacional, el tratamiento para la TBC, ya sea pulmonar o extrapulmonar, se realiza siguiendo el esquema primario para casos nuevos, con o sin confirmación bacteriológica, el cual es: 2HRZE/4H3R3, fase inicial de dos meses con isoniacida $(H)$, rifampicina $(R)$, pirazinamida $(Z)$ y etambutol $(E)$, en forma diaria y posteriormente fase de continuación de cuatro meses con $\mathrm{H}$ y R, tres veces por semana ${ }^{48}$.

Este tratamiento es curativo; sin embargo, en la TBC peritoneal son factores de mal pronóstico el retardo en el diagnóstico, la existencia de enfermedades asociadas y la resistencia a fármacos ${ }^{23}$.

Aunque los corticoesteroides parecen tener algún beneficio en pacientes (prevención de estenosis sintomática y obstrucción intestinal), la calidad deficiente de los estudios limita la generalización de sus hallazgos ${ }^{49}$. Por otra parte, el tratamiento quirúrgico debe reservarse para aquellos casos que cursen con complicaciones, tales como: obstrucción, perforación, fístulas y sangrado gastrointestinal $^{19}$.

\section{Conclusión}

La TB peritoneal, aunque es de escasa ocurrencia en pediatría, es una forma grave de TBC extrapulmonar. 
Constituye un desafío diagnóstico dada la variabilidad e inespecifidad de su presentación. El caso clínico aquí presentado tuvo una mayor dificultad diagnóstica ya que no había antecedentes familiares de TBC o personas con síntomas respiratorios crónicos, como tampoco condición de inmunosupresión, lo que lo constituye en un caso aún más excepcional. De esta manera, es importante su sospecha diagnóstica en un paciente con fiebre, ascitis y/o masa abdominal o pélvica, para así lograr un diagnóstico precoz y de esta manera disminuir la morbimortalidad asociada.

Agradecimientos: Los autores agradecen a la infectóloga Dra. Leonor Jofré M. quien colaboró en la revisión del manuscrito y al médico radiólogo Jaime Verdugo B. quien contribuyó en el informe de las imágenes.

\section{Resumen}

La peritonitis tuberculosa es una entidad infrecuente en la población pediátrica. Es una forma poco común de tuberculosis extrapulmonar y representa un muy bajo porcentaje de todos los casos de tuberculosis. Sus síntomas son inespecíficos, manifestándose usualmente con ascitis, dolor abdominal, fiebre y baja de peso. El retraso en su diagnóstico y tratamiento, dada su forma de presentación, puede incrementar su morbimortalidad. Se comunica el caso de una adolescente de 14 años, previamente sana, quien se presentó con fiebre y ascitis. La laparoscopia demostró múltiples nódulos en la cavidad abdominal compatibles con una tuberculosis peritoneal, la cual fue posteriormente confirmada por cultivo y biología molecular. La paciente completó su tratamiento antituberculoso recuperándose en forma satisfactoria.

\section{Referencias bibliográficas}

1.- Programa Nacional de Control y Eliminación de la Tuberculosis. Informe de Situación 2016. Ministerio de Salud 2017. Disponible en: https://www.minsal.cl/sites/default/files/ NORMA_TECNICA_TUBERCULOSIS.pdf.

2.- Herrera T. La tuberculosis infantil en Chile. Rev Chil Pediatr 2017; 88: 449-50. doi: $10.4067 / \mathrm{S} 0370-41062017000400001$.

3.- Peto HM, Pratt RH, Harrington TA, LoBue PA, Armstrong LR. Epidemiology of extrapulmonary tuberculosis in the United States, 1993-2006. Clin Infect Dis 2009; 49:1350-7. doi: 10.1086/605559.

4.- Rathi P, Gambhire P. Abdominal tuberculosis. J Assoc Physicians India 2016; 64: 38-47.

5.- Zaslavsky J, Mulugeta-Gordon L, Vasko I, Presenza T, Scattergood E, Meislich D, et al. Tuberculous peritonitis in children: Two case reports highlighting the important role of imaging. Radiol Case Rep. 2018; 13: 862-6. doi: 10.1016/j.radcr.2018.05.010.

6.- Tinsa F, Essaddam L, Fitouri Z, Brini I, Douira W, Ben Becher S, et al. Abdominal tuberculosis in children. J Pediatr Gastroenterol Nutr 2010; 50: 634-8. doi: 10.1097/ MPG.0b013e3181b6a57b.

7.- Sanai F M, Bzeizi K I. Systematic review: tuberculous peritonitis-presenting features, diagnostic strategies and treatment. Aliment Pharmacol Ther 2005; 22: 685-700. doi: 10.1111/j.1365-2036.2005.02645.x

8.- Khan F Y, Al-Muzrakchi A M, Elbedawi M M, Al-Muzrakchi A A, Al Tabeb A. Peritoneal tuberculosis in Qatar: A five-year hospital-based study from 2005 to 2009 . Travel Med Infect Dis 2012; 10: 25-31. doi: 10.1016/j. tmaid.2011.12.001.
9.- Kocaman O, Danalioğlu A, İnce A T, Tozlu M, Şentürk H. Diagnosis of tuberculous peritonitis using endoscopic ultrasound-guided fine-needle aspiration biopsy of the peritoneum. Turk J Gastroenterol 2013; 24: 65-9. doi: 10.4318/ tjg.2013.0543.

10.- Avcu G, Sensoy G, Karli A, Caltepe G, Sullu Y, Belet N, Bilgici M C. A case of tuberculous peritonitis in childhood. J Infect Public Health 2015; 8: 369-72. doi: 10.1016/j. jiph.2015.03.004.

11.- Gürkan F, Ozateş M, Boşnak M, Dikici B, Boşnak V, Taş M A, et al. Tuberculous peritonitis in 11 children: clinical features and diagnostic approach. Pediatr Int 1999; 41: 5103 .

12.- Guirat A, Koubaa M, Mzali R, Abid B, Ellouz $\mathrm{S}$, Affes N, et al. Peritoneal tuberculosis. Clin Res Hepatol Gastroenterol 2011; 35: 60-9. doi: 10.1016/j.gcb.2010.07.023.

13.- Chow K M, Chow V C, Hung L C, Wong S M, Szeto C C. Tuberculous peritonitis-associated mortality is high among patients waiting for the results of mycobacterial cultures of ascitic fluid samples. Clin Infect Dis 2002; 35: 409-13.

14.- Forssbohm M, Zwahlen M, Loddenkemper R, Rieder H L. Demographic characteristics of patients with extrapulmonary tuberculosis in Germany. Eur Respir J 2008; 31: 99-105. doi: 10.1183/09031936.00020607.

15.- Ndoye N A, Mbaye P A, Tendeng J N, Cissé L, Diao M L, Dieng M, et al. Peritoneal tuberculosis in infants: diagnostic challenges. European J Pediatr Surg Rep 2018; 6: e87-e89. doi: 10.1055/s-0038-1675406.

16.- Dinler G, Sensoy G, Helek D, Kalayci A G. Tuberculous peritonitis in children: report of nine patients and review of the literature. World J Gastroenterol 2008; 14: 7235-9. doi: 10.3748/ wjg.14.7235

17.- Lin Y S, Huang Y C, Lin T Y. Abdominal tuberculosis in children: a diagnostic challenge. J Microbiol Immunol Infect 2010; 43:188-93. doi: 10.1016/S1684-1182(10)60030-8.

18.- Khan F Y. Peritoneal tuberculosis: advances and controversies. Libyan J Med Sci 2018; 2: 3-7. doi: 10.4103/LJMS.LJMS 3517.

19.- Vaid U, Kane G C. Tuberculous peritonitis. Microbiol Spectr 2017; 5 (1). doi: 10.1128/ microbiolspec.TNMI7-0006-2016.

20.- Mimidis K, Ritis K, Kartalis G. Peritoneal tuberculosis. Ann Gastroenterol 2005; 18:3259.

21.- Malikowski T, Mahmood M, Smyrk T, Raffals L, Nehra V. Tuberculosis of the gastrointestinal tract and associated viscera. $\mathrm{J}$ Clin Tuberc Other Mycobact Dis 2018; 12: 1-8. doi: 10.1016/j.jctube.2018.04.003.

22.- Malik R, Srivastava A, Yachha SK, Poddar U, Lal R. Childhood abdominal tuberculosis: disease patterns, diagnosis, and drug resistance. Indian J Gastroenterol 2015; 34: 418-25. doi: 10.1007/s12664-015-0582-3.

23.- Esposito S, Bosis S, Canazza L, Tenconi R, Torricelli M, Principi N. Peritoneal tuberculosis due to multidrug-resistant Mycobacterium tuberculosis. Pediatr Int 2013; 55: e20-2. doi: 10.1111/j.1442-200X.2012.03735.x.

24.- Silva R, Jara J, Soto T, Sepúlveda P. Severe disseminated tuberculosis in a patient on immunosuppressive treatment. Report of one case. Rev Med Chil 2011; 139: 774-8. doi: / S0034-98872011000600012.

25.- Rasheed S, Zinicola R, Watson D, Bajwa $\mathrm{A}, \mathrm{McDonald}$ PJ. Intra-abdominal and gastrointestinal tuberculosis. Colorectal Dis 2007; 9: 773-83. doi: 10.1111/j.14631318.2007.01337.x 
26.- Farías-Llamas O A, López M K, Morales J M, Medina M. Tuberculosis peritoneal e intestinal: una enfermedad ancestral que impone nuevos retos en la era tecnológica. Informe de un caso y revisión de la literatura. Rev Gastro Enterol Mex 2005; 70:169-78.

27.- Simsek H, Savas M C, Kadayifci A, Tatar G. Elevated serum CA 125 concentration in patients with tuberculous peritonitis: a case control study. Am J Gastroenterol 1997; 92: 1174-6.

28.- Choi C H, Kim C J, Lee Y Y, Kim J S, Song T, Park H S, et al. Peritoneal tuberculosis: a retrospective review of 20 cases and comparison with primary peritoneal carcinoma. Int J Gynecol Cancer 2010; 20: 798-803.

29.- Charoensak A, Nantavithya P, Apisarnthanarak P. Abdominal CT findings to distinguish between tuberculous peritonitis and peritoneal carcinomatosis. J Med Assoc Thai 2012; 95: 1449-56.

30.- Palmer K R, Patil D H, Basran G S, Riordan J F, Silk D B. Abdominal tuberculosis in urban Britain-a common disease. Gut 1985; 26: 1296305.

31.- Arias Guillén M. Advances in the diagnosis of tuberculosis infection. Arch Bronconeumol 2011; 47: 521-30. doi: 10.1016/j. arbres.2011.06.018.

32.- Suntur B M, Kuşçu F. Pooled analysis of 163 published tuberculous peritonitis cases from Turkey. Turk J Med Sci 2018; 48: 311-17. doi: 10.3906/sag-1701-32.

33.- Pereira J M, Madureira A J, Vieira A, Ramos I. Abdominal tuberculosis: imaging features. Eur J Radiol 2005; 55: 173-80. doi: 10.1016/j. ejrad.2005.04.015.

34.- Contardo V, Cofré J, Hernández P. Mal de Pott y diagnóstico inmunológico de tuberculosis, a propósito de un caso pediátrico. Rev Chilena Infectol 2015; 32: 105-10 doi: 10.4067/S071610182015000200015 .

35.- Ge L, Ma J C, Han M, Li J L, Tian J H. Interferon- $\gamma$ release assay for the diagnosis of latent Mycobacterium tuberculosis infection in children younger than 5 years: a meta-analysis. Clin Pediatr (Phila) 2014; 53: 1255-63. doi: 10.1177/0009922814540040.

36.- Sollai S, Galli L, de Martino M, Chiappini E. Systematic review and meta-analysis on the utility of Interferon-gamma release assays for the diagnosis of Mycobacterium tuberculosis infection in children: a 2013 update. BMC Infect Dis 2014; 14 (Suppl 1): S6. doi: 10.1186/1471-2334-14-S1-S6.

37.- Rufai S B, Singh S, Singh A, Kumar P, Singh J, Vishal A, et al. Performance of Xpert MTB/ RIF on ascitic fluid samples for detection of abdominal tuberculosis. J Lab Physicians 2017; 9: 47-52. doi: 10.4103/0974-2727.187927.

38.- Flores L S, Solís A H, Gutiérrez A E, José L C, Ortiz I C, González H G, et al. Peritoneal tuberculosis: A persistent diagnostic dilemma, use complete diagnostic methods. Rev Med Hosp Gen Méx 2015; 78: 55-61. doi: 10.1016/j. hgmx.2015.03.009.

39.- Manual operativo. Implementación del GeneXpert MTB/ RIF en el Programa de Tuberculosis. Programa de Control y Eliminación de la Tuberculosis 2017. Disponible en: https://diprece. minsal.cl/wrdprss_minsal/wp-content/ uploads/2018/02/2018.01.23_MANUALXPERT.pdf.

40.- Lawn S D, Kerkhoff A D, Vogt M, Ghebrekristos Y, Whitelaw A, Wood R. Characteristics and early outcomes of patients with Xpert MTB/ RIF-negative pulmonary tuberculosis diagnosed during screening before antiretroviral therapy. Clin Infect Dis 2012; 54: 1071-9. doi: 10.1093/cid/cir1039.

41.- Ahmad R, Changeez M, Khan J S, Qureshi U, Tariq M, Malik S, et al. Diagnostic accuracy of peritoneal fluid GeneXpert in the diagnosis of intestinal tuberculosis, keeping histopathology as the gold standard. Cureus 2018; 10: e3451. doi: 10.7759/cureus.3451.

42.- Álvarez-Uria G, Azcona J M, Midde M, Naik PK, Reddy S, Reddy R. Rapid diagnosis of pulmonary and extrapulmonary tuberculosis in HIV-infected patients. Comparison of LED fluorescent microscopy and the GeneXpert MTB/RIF assay in a district hospital in India. Tuberc Res Treat 2012; 2012: 932862. doi: $10.1155 / 2012 / 932862$

43.- Abaylı B, Gençdal G. A single-center experience: the diagnostic role of peritoneoscopy in patients with exudative ascites. J Laparoendosc Adv Surg Tech A 2019; 29: 51-4. doi: 10.1089/lap.2018.0274.

44.- Singh M M, Bhargava A N, Jain KP. Tuberculous peritonitis. An evaluation of pathogenetic mechanisms, diagnostic procedures and therapeutic measures. $\mathrm{N}$ Engl $\mathrm{J}$ Med 1969; 281: 1091-4.

45.- Anderson S T, Kaforou M, Brent A J, Wright V J, Banwell C M, Chagaluka G, et al. Diagnosis of childhood tuberculosis and host RNA expression in Africa. N Engl J Med 2014; 370 : 1712-23. doi: 10.1056/NEJMoa1303657.

46.- Lataifeh I, Matalka I, Hayajneh W, Obeidat B, Al Zou'bi H, Abdeen G. Disseminated peritoneal tuberculosis mimicking advanced ovarian cancer. J Obstet Gynaecol 2014; 34 : 268-71. doi: 10.3109/01443615.2013.870140.

47.- Wang S B, Ji Y H, Wu H B, Wang Q S, Zhou W L, Lv L, et al. PET/CT for differentiating between tuberculous peritonitis and peritoneal carcinomatosis: the parietal peritoneum. Medicine (Baltimore) 2017; 96: e5867. doi: 10.1097/MD.0000000000005867.

48.- Ministerio de Salud. Normas técnicas para el control y la eliminación de la tuberculosis. Programa nacional para el control y la eliminación de la tuberculosis. Santiago de Chile - 2014. Disponible en: https:// www.minsal.cl/sites/default/files/NORMA TECNICA TUBERCULOSIS.pdf. Accedido: 26 de marzo 2019.

49.- Soni H, Bellam B L, Rao R K, Kumar P M, Mandavdhare H S, Singh H, et al. Use of steroids for abdominal tuberculosis: a systematic review and meta-analysis. Infection 2019; 47: 387-94. doi: 10.1007/s15010-018$1235-0$. 